\title{
A Review on Wearable and Contactless Sensing for COVID-19 With Policy Challenges
}

\author{
Sagar Suresh Kumar ${ }^{1 *}$, Kia Dashtipour ${ }^{1}$, Qammer H. Abbasi ${ }^{1,2}$, Muhammad A. Imran ${ }^{1,3}$ \\ and Wasim Ahmad ${ }^{\text {1* }}$
}

1 James Watt School of Engineering, University of Glasgow, Glasgow, United Kingdom, ${ }^{2}$ Queen Mary University of London, England, United Kingdom, ${ }^{3}$ Artificial Intelligence Research Center (AIRC), Ajman University, Ajman, United Arab Emirates

\section{OPEN ACCESS}

Edited by:

Syed Ali Raza Zaidi,

University of Leeds, United Kingdom

Reviewed by:

Syed Aziz Shah,

Coventry University, United Kingdom

Qasim Zeeshan Ahmed,

University of Huddersfield,

United Kingdom

Xiaodong Yang

Xidian University, China

*Correspondence:

Sagar Suresh Kumar

sagar.suresh09@gmail.com

Wasim Ahmad

Wasim.Ahmad@glasgow.ac.uk

Specialty section:

This article was submitted to

IoT and Sensor Networks,

a section of the journal

Frontiers in Communications and

Networks

Received: 01 December 2020 Accepted: 29 March 2021

Published: 19 May 2021

Citation:

Suresh Kumar S, Dashtipour K, Abbasi QH, Imran MA and Ahmad W (2021) A Review on Wearable and Contactless Sensing for COVID-19 With Policy Challenges.

Front. Comms. Net. 2:636293. doi: 10.3389/frcmn.2021.636293
The COVID-19 pandemic has affected more than 100 million people worldwide, with around 500,000 cases reported daily. This has led to the overwhelming of healthcare systems even in developed countries such as the US, UK, etc. Remote monitoring of COVID-19 patients with non-serious symptoms can help reduce the burden on healthcare facilities and make them available for high risk groups and the seriously affected. The pandemic has accelerated the demand for the remote patient monitoring (RPM) technologies, and the market is expected to reach 2.14 billion in 2027 from the value of 786.4 million in 2019. In RPM programs, there are two types of sensors that can be used: wearable and contactless. The former, which is currently more widely used, is not only more obtrusive and uncomfortable, but can also lead to cross-infection through patient contact. These two types of technologies are discussed and compared for each vital sign. In the respiratory system, the vital signs are the respiratory rate (RR) and oxygen saturation (SpO2), while for the latter, they are the heart rate/rhythm and the blood pressure (BP). Then, the discussion is broadened to policy level changes needed to expedite the use of such technologies for remote patient monitoring (RPM) in the world. Around $80 \%$ of countries' RPM programs are either informal or in a pilot phase, and thus lack policies and an established regulatory framework to implement their programs. The various policies needed to initiate, deliver, and reimburse RPM programs during emergency situations and outbreaks are discussed. Finally, technologies such as contactless systems, robotics, and Internet-of-things (IOT) that will revolutionize healthcare in the future by reducing the interaction between physicians and patients and cross-infection are discussed.

\begin{abstract}
Keywords: COVID-19, coronavirus, remote patient monitoring, contactless physiological sensors, wearable physiological sensors, camera-based physiological measurement, radar-based physiological measurement, telehealth
\end{abstract}

\section{INTRODUCTION}

The COVID-19 pandemic, caused by the novel coronavirus SARS-CoV-2, has spread to over 200 countries across the globe with around 100 million cases and 2 million deaths (WHO, 2020a). The health systems of several countries including those with a high quality of healthcare such as the US have been overwhelmed due to an excessive patient influx (Mareiniss, 2020). The disease manifests differently in people from a mild or asymptomatic form to a critical one. Only those who are critically ill and/or in need of external ventilation are hospitalized, and those with milder 


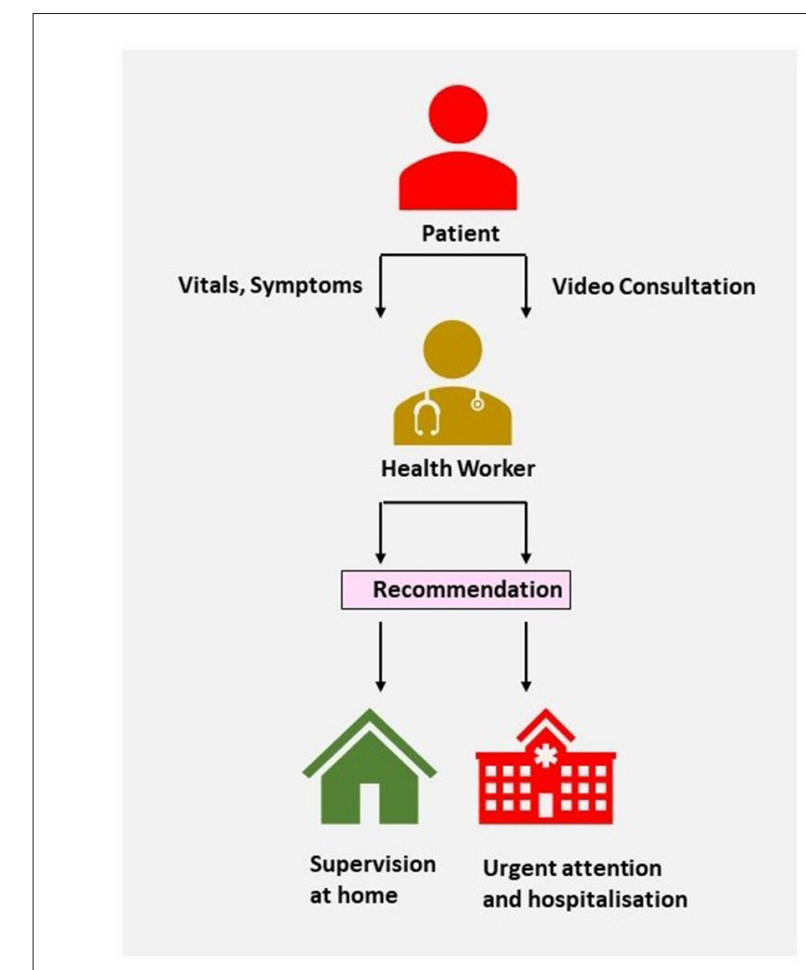

FIGURE 1 | A typical patient monitoring system.

symptoms are told to isolate at home as long as their condition does not deteriorate (WHO, 2020c). These steps prevent the overloading of health systems.

With regards to the above, Remote Patient Monitoring (RPM) may have several benefits. It helps monitor vulnerable groups, i.e., the elderly or those with pre-existing health conditions such as cardiovascular and respiratory disorders (WHO, 2020b). In addition, patients with medical issues other than COVID-19 would not be required to visit hospitals, thereby reducing risks of cross-infection. Furthermore, such schemes are quite viable given the recent developments in the fields of Point-of-care (POC) sensors, Internet-of-things(IoT), and telemedicine. The pandemic has accelerated the demand for the RPM technologies, and the market is expected to reach 2.14 billion in 2027 from the value of 786.4 million in 2019 (Anonymous, 2020). Countries such as the UK are actively looking for innovative operating models that include telemedicine and RPM technologies to recover from the pandemic (Crouch, 2020). Thus, it can be expected in the future that healthcare will become more personalized. Recently, the United Kingdom National Health Service (NHS) provided patients recovering from cystic fibrosis and COVID-19 with wearable devices combined with software applications for RPM. The Precision Recovery Program (PRP) is an RPM initiative of the Mount Sinai Health System spanning across its eight hospitals in the US in which patients are referred if they display the symptoms of COVID-19 (Laura Tabacof and Putrino, 2020). A typical patient monitoring system is illustrated in Figure 1.
RPM sensors can be broadly categorized into two types, wearable and contactless. Although wearable sensors are more widely used currently, contactless ones have certain advantages over them. Generally, they are less intrusive and more comfortable. This is especially important for the elderly and/or physically compromised, who may not be able to wear multiple sensors for a long time (Gjoreski and Gams, 2011). Secondly, this will reduce the risks of infection for doctors and health care workers as contact is minimized. The consulting firm Frost and Sullivan reported that patient monitoring technologies are moving away from wearable sensors toward the use of video, sound, radar, and other contactless methods in conjunction with machine learning and artificial intelligence technologies (Fernandez, 2020).

Several innovative sensors and sensing technologies related to healthcare have been developed in the past few years. Instead of just focusing on these novel wearable and contactless technologies individually like other review studies (Islam et al., 2020; Taylor et al., 2020), we compare their performance and feasibility with the conventional methods for each vital sign. This should help guide researchers, physicians and healthcare workers to choose suitable technologies depending on patient requirements and environmental conditions. Also, review papers till now have only focused on the technical aspects of sensors mostly from academia, but did not consider the wider non-technical challenges in implementing RPM programs across different nations. Thus, this study further addresses the policy level challenges to such RPM programs. Finally, this study discusses the future directions and technologies that will transform the field of healthcare by making it more personalized and reducing patient-physician interactions, and thus cross-infection.

The rest of this paper is organized as follows. Several wearable/contact and contactless sensor technologies that monitor vital signs, such as respiratory rate, oxygen saturation, heart rate, etc., including their feasibility and practical limitations are discussed in section 2. The policy challenges faced by many developed countries in the implementation of RPM and other tele-health programs are discussed in section 3. After discussing the future directions in section 4 , the paper is concluded in section 5 .

\section{SENSING TECHNOLOGIES}

\subsection{Respiratory System}

COVID-19 is a disease that primarily affects the respiratory tract and can cause the lungs to fill with fluid and get inflamed. This affects oxygen uptake and leads to hypoxia (Shi et al., 2020). The two vital signs to assess the condition of the respiratory tract are discussed in the following sections.

\subsubsection{Respiratory Rate}

Respiratory rate (RR) is defined as the mean number of breaths drawn per minute, and that of 30 breaths/minute or greater is a sign of severe pneumonia, a principal symptom of COVID19 (Contributors, 2020). There are, broadly, two methods of measuring RR-(1) airflow sensors using temperature, humidity, 
etc. changes, (2) respiratory effort detection using chest and abdominal movements, breathing sounds, cough, etc. Wearable airflow methods typically involve placing an airflow sensor in the proximity of the mouth. Rangel and Castillo (2020) placed an airflow sensor in a mask to detect the heat generated by breathing. Such a mask based design can be quite useful during the pandemic. Among effort based techniques, Elfaramawy et al. (2019) designed a system that uses nine axial inertial measurement units (IMUs) on the chest and abdomen to calculate the respiratory rate as well as a microphone for cough detection. Imran et al. (2020) developed an AI powered solution that distinguishes COVID-19 related coughs from Non COVID19 coughs. It is able to be deployed as a smartphone app that records 3 cough sounds and returns the result within $2 \mathrm{~min}$. Other than intrusiveness, wearable sensors for RR are quite sensitive to motion artifacts (Fedotov et al., 2018).

In contactless methods, microphones placed in the proximity of the patient's body but not so close to the mouth have been used to detect respiratory sounds (Azam et al., 2018), although they may be susceptible to external sounds from the environment. One advantage is that these methods can be implemented by using only a smartphone microphone. The second way involves the use of cameras to track the motion of the chest wall. Massaroni et al. (2018) used a commercial RGB camera to calculate the respiratory rate by analyzing the variations in the intensity of reflected light created by chest motion. Wang et al. (2020) used Microsoft Kinect cameras to take depth images, after which a deep learning framework was used to classify abnormal breathing patterns associated with COVID-19. This approach obtained an accuracy of $94.5 \%$. Even though these methods have been benefited by the recent advancements in image processing and $\mathrm{AI}$, the price of such cameras and privacy issues are major obstacles (Elphick et al., 2013). Ultrasound (US) techniques rely on transmitting and receiving high frequency sound waves to and from the breathing body. They have been implemented using portable smartphone-based systems, which just like camera technology, can be integrated with deep learning techniques and convolutional neural networks (Taylor et al., 2020). One obstacle to continuous monitoring is that the patients need to prepare themselves for the US scan and one of the steps involves not eating a few hours before.

Radar systems can monitor RR by using frequency-modulated continuous-wave (FMCW) to observe Doppler shifts associated with breathing movements, and have been able to obtain accuracy of 99\% (Tsai et al., 2020). FMCW radar works by transmitting frequency modulated pulses, i.e., pulses with periodic shifts in frequency, and the received echos undergo respective frequency shifts due to the motion of the body, known as the Doppler effect. Different frequency bands can be used and the sensitivity normally increases with frequency. Yang et al. used 5G wireless networks to transmit breathing information from a C-band $(4-8 \mathrm{~Hz})$ system, and obtained accuracies above 98\% (Yang et al., 2019). This system obtained a transmission speed of 3,500 packets/second which should enable high speed RPM programs. In their later paper, an S-band $(2-4 \mathrm{GHz})$ system was used to detect the shallow breathing associated with hypopnea (Yang et al., 2020). Israel recently adapted military radar systems for contactless monitoring of COVID-19 patients in hospitals (Ahronheim, 2020). Their Defense Ministry intends to adapt more of such technologies intended for military and/or security purposes for health monitoring, which seems to be an innovative and cost-effective strategy. However, the major drawback of radar systems is the high power requirement, which affects the feasibility and cost of such solutions for continuous monitoring. The range can also be an issue as the patient would need to be within a certain distance from the device for it to work (Fletcher and Jing Han, 2009).

\subsubsection{Oxygen Saturation}

Oxygen Saturation (SpO2) is the proportion of oxygen-saturated hemoglobin to the total hemoglobin levels. The Pulseoximeter is the standard medical device to calculate the $\mathrm{SpO} 2$, and is usually worn over the index finger. The device works by analyzing the difference in absorption of two wavelengths of light by oxygenated and deoxygenated hemoglobin, known as the photoplethysmogram (PPG) signal. Oxitone medical's wrist worn pulseoximeter received clearance from the US Food and Drug Administration (FDA), and they plan to integrate it with their digital health platform capable of giving alerts and analytics (Comstock, 2020). Recently, smartphone based alternatives have been proposed that use the in-built optical sensor (Scully et al., 2011). In Ma et al. (2019)'s study, a mobile phone was sufficient to perform the complete pre-processing of PPG data including filtering. Since these devices need to be placed over the index finger correctly and have proper contact with the skin to work, elderly people may require the assistance of health professionals. Casalino et al. (2020) proposed a contact free RGB camera based strategy that processes video frames of a person's face to find the changes in the absorption of light, and hence the $\mathrm{SpO}$. It is both contact-free and easy to use and can run on any mobile device with a camera such as a smartphone or laptop. In the design, the forehead and 2 cheeks are taken as regions of interest (ROI) as they have been found to be the most suitable areas for extracting the PPG signal (Casalino et al., 2020). The signals are processed, filtered, and then, similar to before, the differences in absorption of red and blue light are analyzed.

\subsection{Cardiovascular System}

People with cardiovascular problems are at a higher risk of developing COVID-19. In a study conducted in Wuhan on 191 subjects, 91 (48\%) patients had a co-morbidity, with hypertension or high blood pressure being the most prevalent (58 or 30\% patients). Therefore, there is a need to identify such high risk groups (Zheng et al., 2020). In addition, the disease damages the cardiovascular system and leads to myocardial injury. In the study, the National Health Commission of China (NHC) reported that among the people who died from COVID-19, $11.8 \%$ people had serious heart damage.

\subsubsection{Heart Rate and Rhythm}

COVID-19 produces discernible changes known as arrhythmias in a person's electrocardiogram (ECG) signal, a measure of the electrical activity of the heart. A typical ECG pulse, illustrated in Figure 2, consists of: P wave representing atrial contraction, 
QRS complex representing ventricular contraction, and $\mathrm{T}$ wave representing ventricular relaxation. The time interval between two successive $\mathrm{R}$ peaks represents the time taken for one beat, and can be used to calculate the heart rate. Some arrhythmias include ventricular tachycardia or an abnormal increase in heart rate, ST-segment elevation, QT prolongation, etc. (Mehraeen et al., 2020). Traditional ambulatory Holter monitoring schemes involve wearing a device with multiple electrodes and lead wires attached to the chest. ECG patch electrodes, which have microelectronic circuits, are a smaller and wireless alternative. They are typically attached to the left chest with a water-proof adhesive and can monitor activity for two or more weeks. VitalPatch was granted an emergency use authorization by the FDA to monitor COVID-19 patients (Muo, 2020). With regards to wrist based alternatives and smart-watches, Samsung's Galaxy Watch 3 has recently received FDA approval for ECG monitoring (Wetsman, 2020). However, both patch electrodes and wristwatches are mostly single channel systems, and they may not be able to detect arrhythmias caused by a change in the QRS axis or QRS width. The higher the number of leads in an ECG system, the more arrhythmias it can detect (Anonymous, 2019). The 12lead ECGs detect the most number of arrhythmias followed by 5-lead systems (Anonymous, 2019). Long Short Term Network (LSTM) deep learning approach helped minimize the effect of noises such as patient motion and baseline wander (Laitala et al., 2020).

Although radar technology can measure the heart rate from chest motion (Nosrati and Tavassolian, 2018), it cannot track arrythmias such as ventricular fibrillation while the ECG can. The PPG is a viable alternative to 1-lead ECG systems but it is still inferior to 5-lead and 12-lead ones in the ability to detect arrhythmias (Anonymous, 2019). Many commercially available smartphone applications that use a phone's camera to obtain the PPG are not as accurate as the clinical ECG and fingertip pulseoximeters, and hence, are not regulated as medical devices (Coppetti et al., 2020). Ha et al. (2020) used a deep learning based radar scheme to obtain the Seismocardiogram (SCG), which is a measure of the mechanical activity of the heart as opposed to electrical. They used radar to record the micro-vibrations of the heart and then reconstructed the SCG waveform from them. This offers additional information such as the opening and closing of valves that is not available using standard radar methods. Another advantage radar has is that it can be used to simultaneously measure the heart rate and respiratory rate, and the two signals can be separated based on their frequencies (Walterscheid et al., 2019). This reduces the need for an additional system and hence, the expenses.

\subsubsection{Blood Pressure}

Traditionally, blood pressure (BP) is monitored using cuff-based devices known as the sphygmomanometer that requires the help of a nurse or a health worker to use. A stethoscope is used to analyze the systole and diastolic sounds, and the respective pressure values, systolic blood pressure (SBP) and diastolic blood pressure (DBP), are noted using the sphygmomanometer. The pulse-transit-time (PTT) method provides an unobtrusive, selfuse, and wireless alternative (Wang et al., 2014). The PTT is

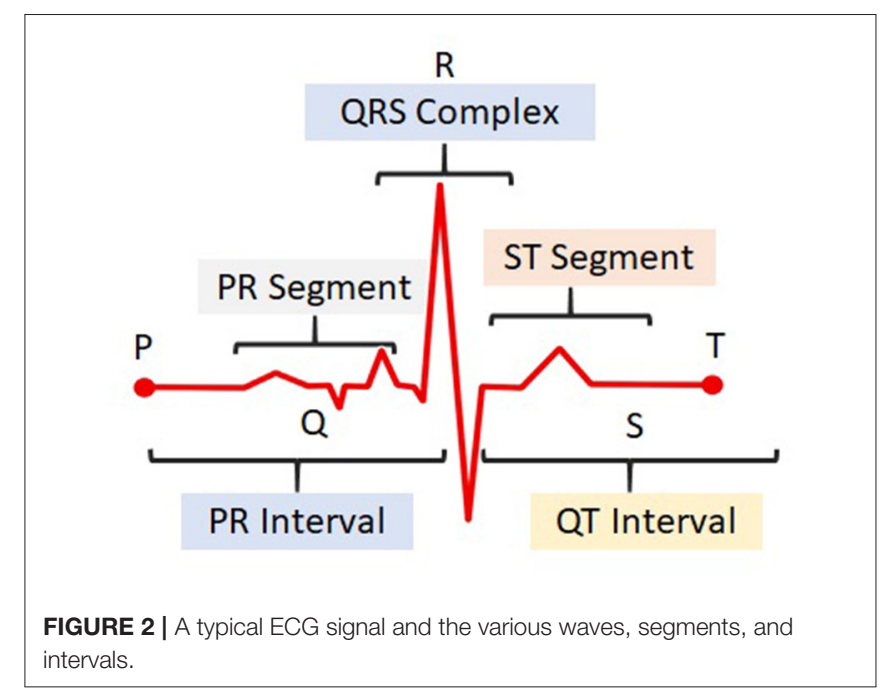

the time taken by the arterial pulse to move from the heart to a peripheral site. It is a measure of arterial stiffness and can be used to find the BP. Previously, PTT was calculated using the difference between the peaks of the ECG R wave and PPG, but now research has shown that it can also be calculated using the difference between PCG signals at two peripheral sites (Huang et al., 2017). This is beneficial for contactless monitoring solutions as an additional modality for $\mathrm{R}$ peak or systole detection such as Radar is no longer required, which reduces the cost of such solutions. Huang et al. (2017) recorded the PPG from the face and the palm to calculate the BP. The extraction of iPPG from cameras is illustrated in Figure 3, which offers a block level description of PPG and BP calculation. Radar technology has also been used for contactless BP monitoring (Ohata et al., 2019). The merits and demerits of different contactless technologies are summarized in Table 1.

\section{POLICY CHALLENGES}

In a global survey on e-Health conducted by the World Health Organization (WHO) on 125 countries, a large number of countries felt that policy level challenges in implementing RPM and other tele-health programs were extremely important compared to other issues, as illustrated in Figure 4 (WHO, 2016). The distribution of tele-health programs across different regions of the world are given in Figure 5. It can be observed that the distribution of RPM programs is uneven, with most in the European and Western Pacific regions and the least in African and South East Asian regions. Also, around 80\% of countries' RPM programs are either informal or in a pilot phase, and thus lack an established regulatory framework to initiate, deliver, and reimburse their programs especially in emergencies such as the pandemic (WHO, 2016). In spite of carrying out tele-health programs for over two decades, developing countries have not achieved much success in reducing the cost and accessibility of care (Bali, 2018). Generally, rural areas, which are more prevalent in developing countries than developed ones, have a 


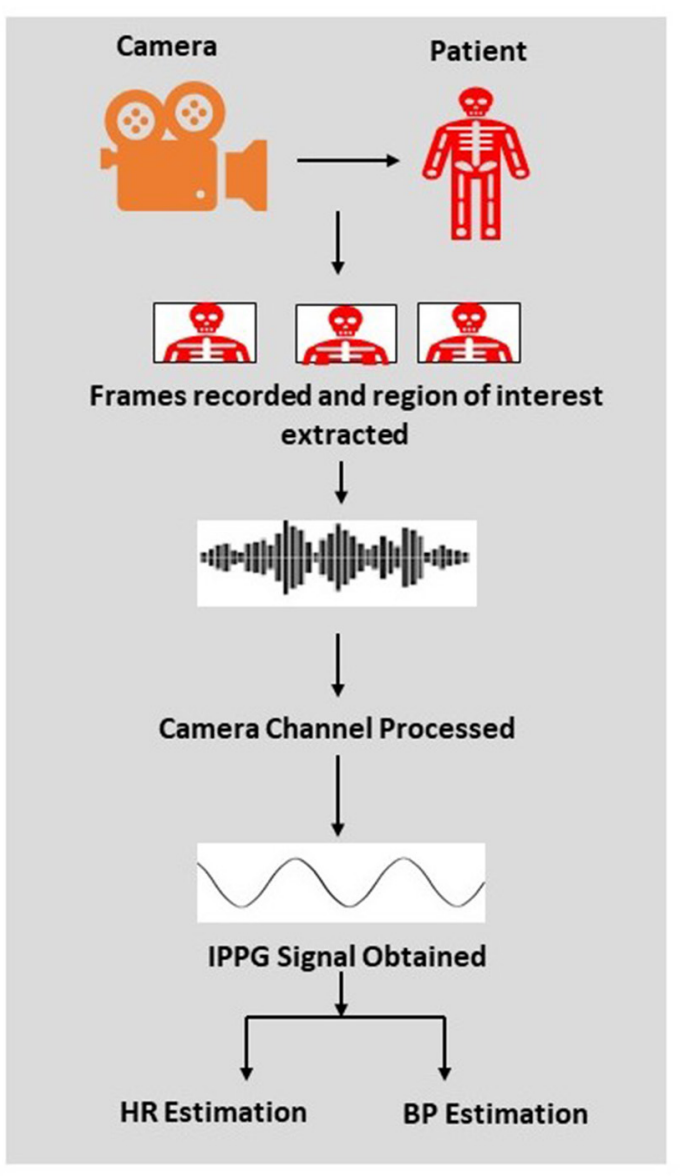

FIGURE 3 | Schematic of iPPG extraction.

TABLE 1 | Different contactless technologies and their advantages and disadvantages.

\begin{tabular}{|c|c|c|}
\hline Technology & Advantage & Disadvantage \\
\hline Microphones & Smartphone sensor is sufficient & $\begin{array}{l}\text { Susceptible to } \\
\text { environmental noise }\end{array}$ \\
\hline Camera & Accurate, Al can be used & $\begin{array}{l}\text { High camera cost, privacy } \\
\text { concerns }\end{array}$ \\
\hline Radar & Accurate, Al can be used & $\begin{array}{l}\text { High cost, high energy } \\
\text { requirements, range issues }\end{array}$ \\
\hline Ultrasound & $\begin{array}{l}\text { Accurate, Al can be used, } \\
\text { portable }\end{array}$ & Patient preparation \\
\hline
\end{tabular}

lack of digital infrastructure compared to urban areas. These countries may also not have the resources to create a centralized digital database of patients and their health status, which is an essential requirement for most tele-health programs (Raza et al., 2018). However, even if the technological disparities are reduced, they cannot be efficiently implemented without guiding policies and frameworks.

The US has recently made significant policy changes, which can be used as a guide by other nations. Current Procedure
Terminology (CPT) codes, which are used by the Centers for Medicare and Medicaid Services (CMS) for the reimbursement of healthcare programs, were updated to allow reimbursement for the following services: reviewing RPM data, consulting patients regarding their RPM data, and training a patient to set up and use RPM technologies (mTelehealth, 2020). The one old code (99091) and three new codes (99453, 99454, 99457), explained in detail in Table 2, will help incorporate the latest RPM technologies. During the pandemic, the CMS made further changes including equal reimbursements for both tele-health and in-person consultations (mTelehealth, 2020). In creating policies and frameworks for swiftly responding to emergencies, communication protocols for handling RPM data are necessary to ensure patient safety and privacy. Clinicians should be able to easily access and discern the large amounts of data coming from RPM as well. Easing regulations required for physiological monitoring devices during such emergencies can help reduce supply shortages. For example, the US Food and Drug Administration(FDA) has relaxed certain regulations, by which devices such as oximeters, apnea monitors, ECG, etc., that were previously approved for hospital use, can now be marketed for home use without any additional procedure (FDA, 2020). Modification of devices to support remote patient monitoring functions without seeking additional approval is also permitted. Finally, public-private partnerships can help countries acquire the latest RPM technologies such as contactless cameras, radar, etc. They have enhanced the viability of tele-health programs in the past, especially in low resource settings (Mahapatra and Sahoo, 2017). All the above-mentioned key factors in choosing RPM technologies are summarized in Figure 6.

\section{FUTURE DIRECTION}

Hospitals and other healthcare organizations around the globe are under immense pressure because of COVID-19. They have to deal with the increased number of infected patients on one hand and control the spread of the infection and protect healthcare providers on the other. Several additional smart sensors and contactless technologies are presented in this section that can collect vital signs remotely and monitor patients from a distance. In the future, smart sensors and contactless technologies will minimize the need for human intervention and will lead to more personalized health systems. Such technological solutions can also improve the overall performance of these systems. Hence, the fourth industrial revolution is expected to revolutionize the field of healthcare as well. A healthcare system in the future might look like Figure 7.

\subsection{Contactless Systems}

As mentioned above, when patients are tested and monitored by healthcare providers, such interactions involve several physical touch-points that increases the risk of healthcare staff being exposed to COVID-19 and other communicable diseases. A number of contactless technologies to reduce physical contact between patients and healthcare workers were mentioned in the previous sections. Additionally, contactless preliminary screening can be performed with a thermal camera that detects 


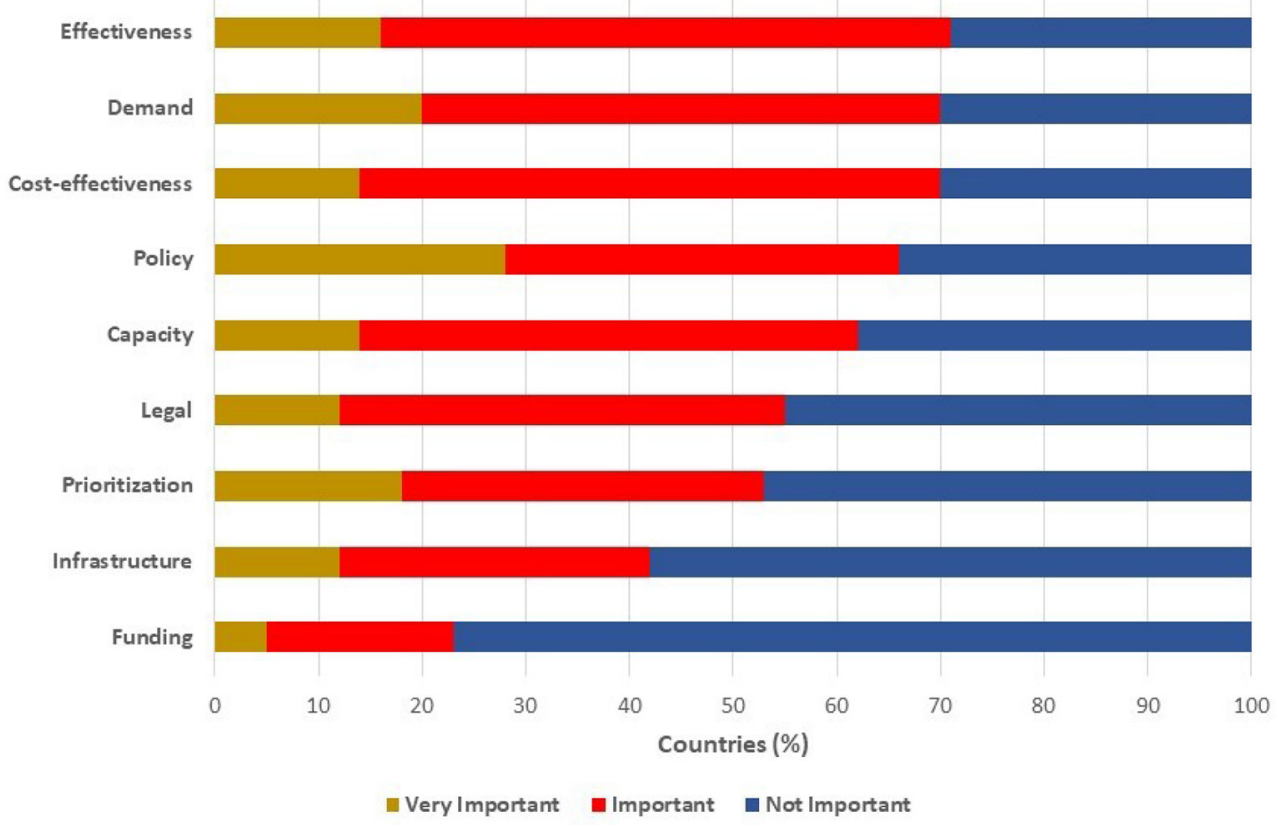

FIGURE 4 | Challenges in implementing tele-health programmes to support UHC, evaluated by level of importance.

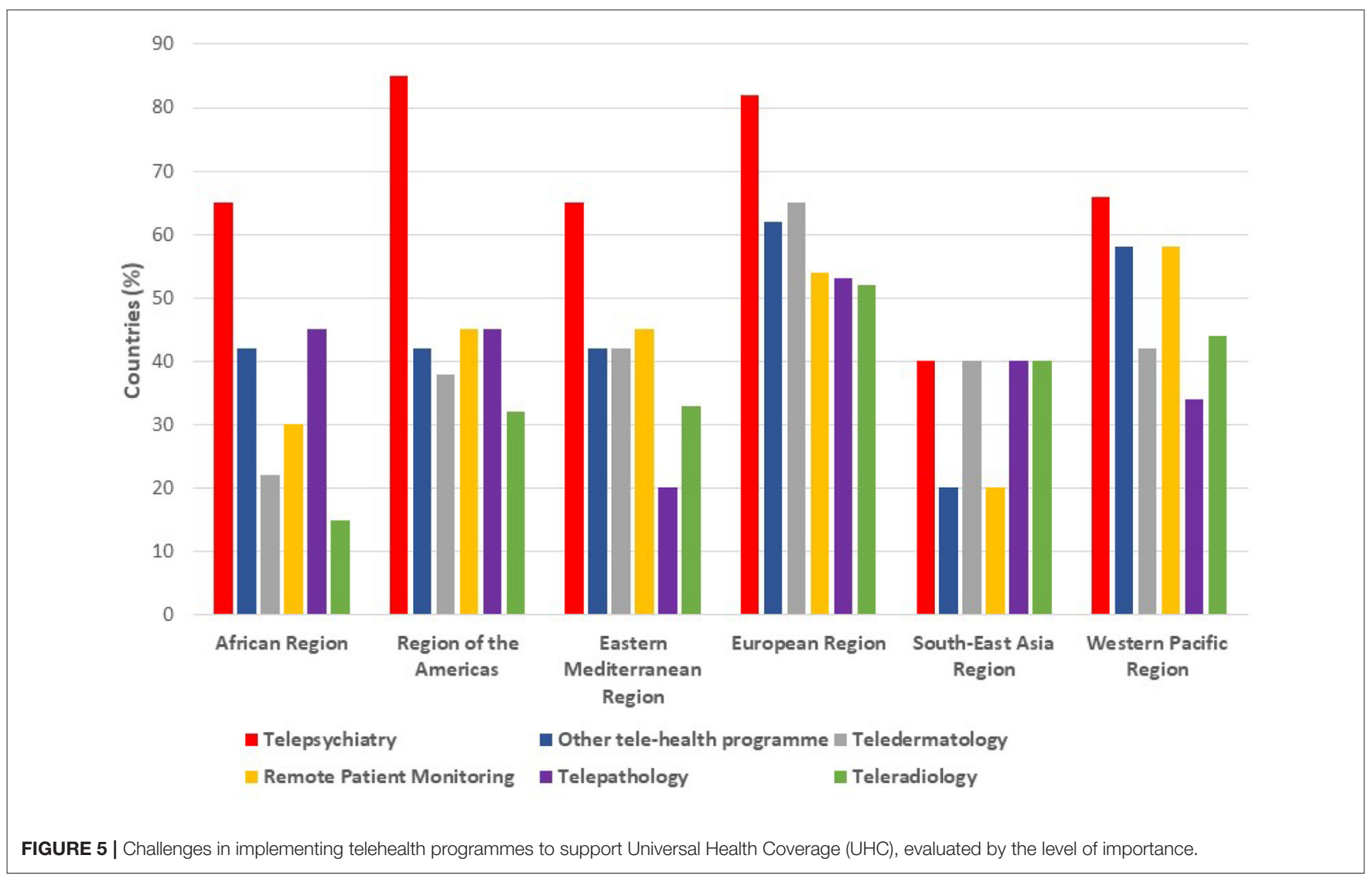


a high temperature. These contactless thermal cameras can serve as first-line screening equipment at hospitals, the workplace, or any other required location. All these technologies can be integrated to form complete contactless care systems to monitor patients at their location- whether in the hospital, outpatient care facility, or home. With the help of efficient alarm systems, the healthcare provider can be notified whenever the patients' health deteriorates and they require urgent attention.

TABLE 2 | Current procedural terminology (CPT) codes for reimbursement of RPM services.

\begin{tabular}{|c|c|}
\hline CPT code & Description \\
\hline 99091 & Collection and interpretation of physiologic data \\
\hline 99453 & Initial set up and patient education on use of equipment \\
\hline 99454 & $\begin{array}{l}\text { Supply of devices, transmission, collection, and report/summary } \\
\text { services to the clinician }\end{array}$ \\
\hline 99457 & $\begin{array}{l}\text { Remote physiological monitoring services for first } 20 \text { min of RPM } \\
\text { services }\end{array}$ \\
\hline 99458 & $\begin{array}{l}\text { Remote physiological monitoring services that exceeds first } 20 \\
\text { min of RPM services }\end{array}$ \\
\hline
\end{tabular}

\subsection{Healthcare Internet of Things (H-loT)}

The healthcare Internet of Things (H-IoT) is the basic enabling technology that facilitates the communication between the smart sensors and the processors at the receiver. Sensors and devices with H-IoT capabilities can help transmit diagnostic information across a large distance, hence minimizing the interaction and close contact between staff and patient without compromising the quality of care. Recent advancements in Blockchain, Cloud and Edge computing have enabled efficient managing, processing and storage of the large volumes of data, known as Big Data, generated by such sensors (Qadri et al., 2020). Blockchain is a decentralized peer-to-peer storage system that stores data in the form of unchangeable blocks. These blocks are made anonymous and unchangeable by assigning them unique hashes that change if the data in the block is changed. This assures the privacy and security of a patient's data. Cloud computing refers to the process of using a connected network of remote servers on the internet to manage and store data rather than local storage resources such as hard disks or local servers. Edge computing reduces the transmission time between the sensors or nodes and processing units by providing computational power locally, toward the edges of the network and closer to the sensors.

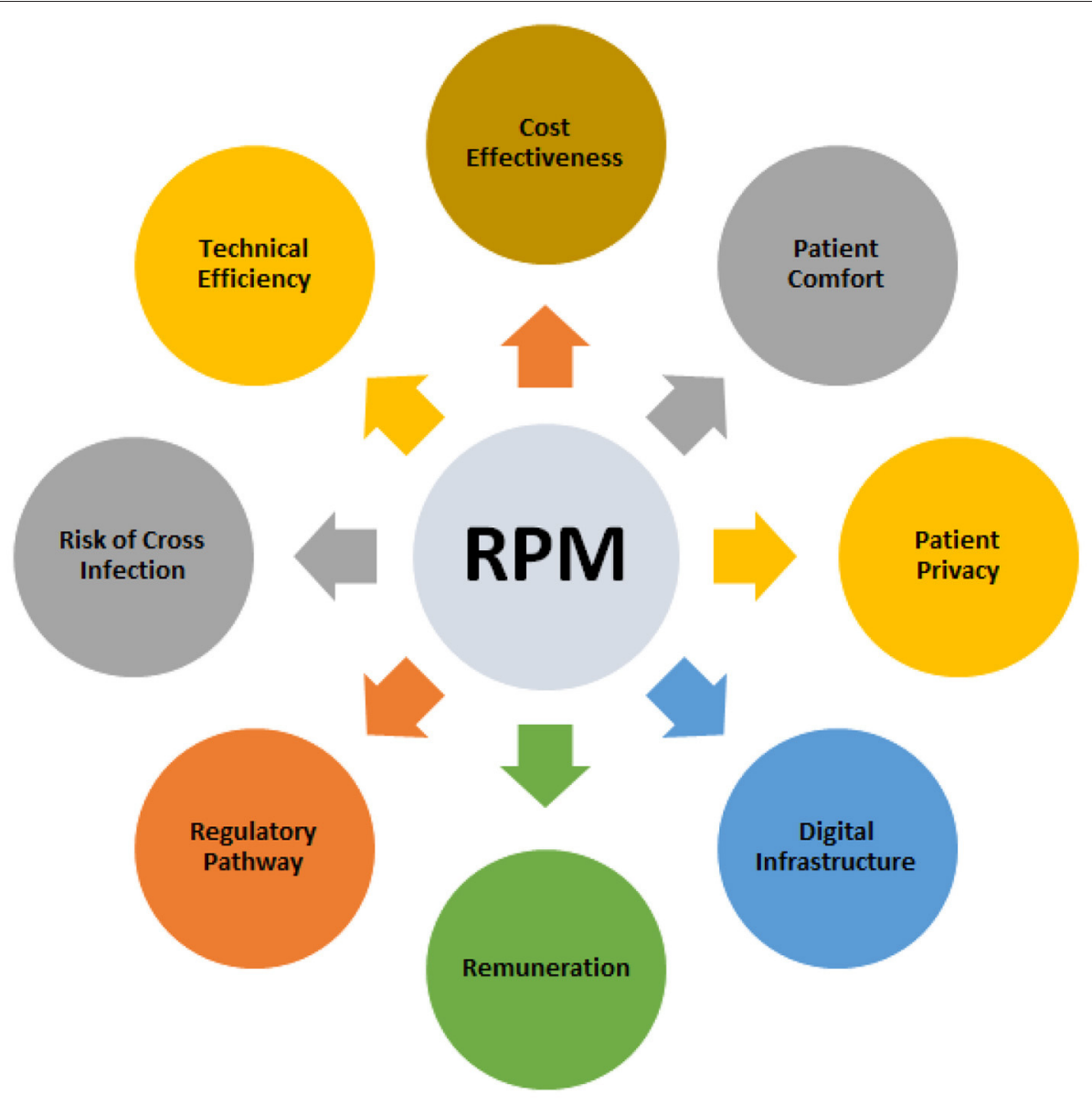

FIGURE 6 | Eight key factors for choosing RPM and sensor technologies. 


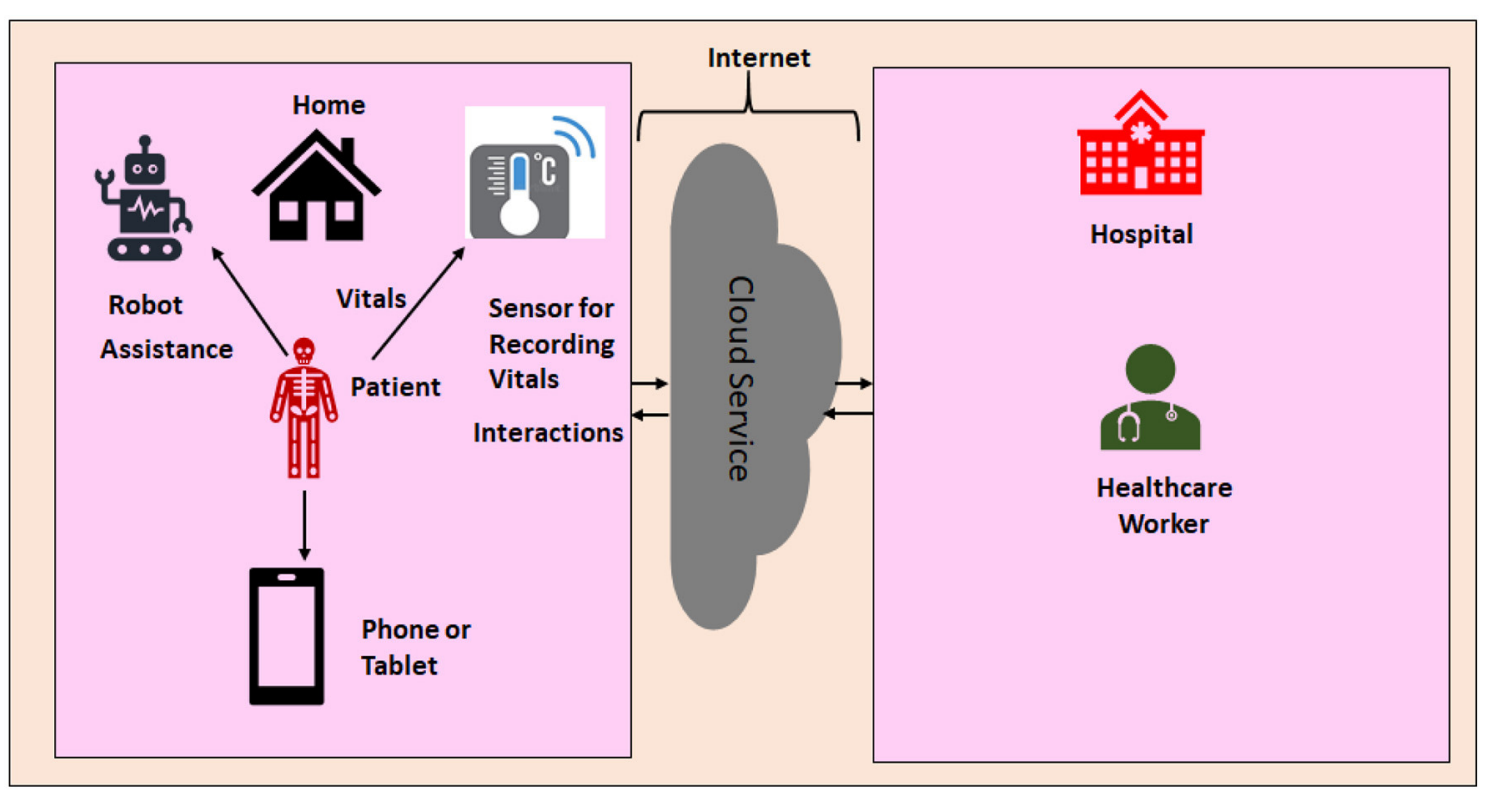

FIGURE 7 | A futuristic healthcare system.

\subsection{Robotics}

Robots and robotic technology can be used to replace people from hazardous jobs. As the spread of the COVID-19 is on the rise, the potential roles of robotics in healthcare and allied areas are becoming increasingly clear. Robots can be deployed for disinfection of the COVID-19 wards, delivering medications and food to the patients, and remotely measuring patients' vital signs. Additionally, there has been a recent interest in using "social robots" to deal with the loneliness faced by older people in assisted living (Pirhonen et al., 2020). As per a survey conducted by Healthwatch North Sommerset, 63\% of elderly and vulnerable people felt isolated and lonely due to the lockdown, with the majority saying this affected their wellbeing and physical health (Angear, 2020). A study led by the University of Genova found that elderly people who spent time with "Pepper," an artificial intelligence powered humanoid robot, reported a notable improvement toward their mental health (Shadwell, 2020). Thus, such solutions are able to address both the physical and mental health issues of patients.

\section{CONCLUSION}

This paper compared the latest wearable and contactless sensor technologies for monitoring the vital signs of the respiratory

\section{REFERENCES}

Ahronheim, A. A. (2020). Radar system protecting Israel's borders will monitor coronavirus patients. Available online at: https://www.jpost.com/israel-news/ radar-system-used-to-protect-israels-border-used-to-monitor-corona-cases623088 and cardiovascular systems of COVID-19 patients remotely and highlighted their merits and shortcoming. To the best of your knowledge, such a comparison is missing in the existing review papers. Among the less obtrusive contactless sensor technologies, visual and camera-based ones seem to be the most prevalent and promising ones, although their price is a major obstacle. Only in cardiac rate and rhythm monitoring, wearable methods (i.e., ECG) may offer a richer diagnosis compare to contactless ones. The discussion then moved to the policy level changes needed to implement RPM programs, which have also been excluded in most studies. Finally, the future of healthcare and potential technologies that can be used to reduce the interaction between healthcare professionals and patients, and hence cross-infection, were discussed. Future work should address the broader field of telehealth rather than just RPM and sensor technologies. The WHO recently released the draft global strategy on digital health 2020-2025, in which there is surely scope for more ideas to deal with pandemics and infectious disease outbreaks (WHO, 2019).

\section{AUTHOR CONTRIBUTIONS}

All authors listed have made a substantial, direct and intellectual contribution to the work, and approved it for publication. SK formulated the theme of this research and chose the topics that were covered. 
Anonymous (2020). Remote patient monitoring market to reach USD 2.14 billion by 2027 | reports and data. Available online at: https://www.bloomberg.com/ press-releases/2020-06-18/remote-patient-monitoring-market-to-reach-usd2-14-billion-by-2027-reports-and-data

Azam, A., Shahzadi, A., Khalid, A., Anwar, S. M., and Naeem, U. (2018). "Smartphone based human breath analysis from respiratory sounds," in 2018 40th Annual International Conference of the IEEE Engineering in Medicine and Biology Society (EMBC) (Honolulu, HI), 445-448. doi: 10.1109/EMBC.2018.8512452

Bali, S. (2018). Barriers to Development of Telemedicine in Developing Countries. doi: 10.5772/intechopen.81723

Casalino, A., Castellano, A., and Zaza, G. (2020). "A mHealth solution for contact-less self-monitoring of blood oxygen saturation," in 2020 IEEE Symposium on Computers and Communications (ISCC) (Rennes), 1-7. doi: 10.1109/ISCC50000.2020.9219718

Comstock (2020). FDA Clears Oxitone's Wrist-Worn Pulse Ox for Remote Patient Monitoring. Comstock.

Contributors, A. (2020). Respiratory management of COVID 19. Physiopedia. Available online at: https://members.physio-pedia.com/learn/respiratorymanagement-of-people-with-covid-19/

Coppetti, T., Brauchlin, A., Müggler, S., Attinger-Toller, A., Templin, C., Schönrath, F., et al. (2020). Accuracy of smartphone apps for heart rate measurement. Eur. J. Prevent. Cardiol. 24, 1287-1293. doi: $10.1177 / 2047487317702044$

Crouch, H. (2020). Government roadmap hints at more use of tele-medicine and remote care. Available online at: https://www.digitalhealth.net/2020/05/ government-roadmap-tele-medicine-remote-care/

Elfaramawy, A., Fall, A., Arab, A., Morissette, M., Lellouche, F., Gosselin, B., et al. (2019). A wireless respiratory monitoring system using a wearable patch sensor network. IEEE Sensors J. 19, 650-657. doi: 10.1109/JSEN.2018.2877617

Elphick, H. E., Alkali, A. H., Kingshott, R. K., Burke, D., and Saatchi, R. (2013). Exploratory study to evaluate respiratory rate using a thermal imaging camera. Respiration 97, 205-212. doi: 10.1159/000490546

FDA (2020). Enforcement Policy for Non-Invasive Remote Monitoring Devices Used to Support Patient Monitoring During the Coronavirus Disease 2019 (COVID19) Public Health Emergency (Revised). FDA.

Fedotov A.A., Akulov S.A., and Akulova A.S. (2018). "Motion artifacts reduction in wearable respiratory monitoring device," in EMBEC \& $N B C$ 2017. EMBEC 2017, NBC 2017. IFMBE Proceedings, Vol. 65, eds H. Eskola, O. Väisänen, J. Viik, and J. Hyttinen (Singapore: Springer). doi: 10.1007/978-981-10-5122-7_280

Fernandez, A. (2020). Advanced non-contact patient monitoring technologies. Available online at: https://ww2.frost.com/news/press-releases/frost-sullivanreveals-non-contact-patient-monitoring-technologies-to-revolutionizehealthcare/

Fletcher, R. and Han, J. (2009). "Low-cost differential front-end for Doppler radar vital sign monitoring," in 2009 IEEE MTT-S International Microwave Symposium Digest (Boston, MA), 1325-1328. doi: 10.1109/MWSYM.2009.5165949

Gjoreski, H., and Gams, M. (2011). "Activity/posture recognition using wearable sensors placed on different body locations," in The Fourteenth International Conference on Artificial Intelligence and Soft Computing, (Crete). doi: 10.2316/P.2011.716-067

Ha, A., Assana, A., and Adib, F. M. (2020). "Contactless seismocardiography via deep learning radars," in The 26th Annual International Conference on Mobile Computing and Networking, Vol. 62 (London, UK: ACM), 1-14. doi: $10.1145 / 3372224.3419982$

Huang, P., Lin, C., Chung, M., Lin, T., and Wu, B. (2017). "Image based contactless blood pressure assessment using pulse transit time," in 2017 International Automatic Control Conference (CACS) (Pingtung), 1-6. doi: 10.1109/CACS.2017.8284275

Imran, A., Posokhova, I., Qureshi, H. N., Masood, U., Riaz, M. S., Ali, K., et al. (2020). AI4COVID-19: Ai enabled preliminary diagnosis for covid19 from cough samples via an app. Inform. Med. Unlocked 20:100378. doi: 10.1016/j.imu.2020.100378

Islam, M., Mahmud, S., Jibril, M., Islam, M., Nooruddin, S., and Ayon, S. (2020). Wearable technology to assist the patients infected with novel coronavirus (COVID-19). SN Comput. Sci. 1:320. doi: 10.1007/s42979-020-00335-4
Laitala, A., Jiang, A., Syrjälä, E., Naeini, E. K., Airola, A., Rahmani, A. M., et al. (2020). "Robust ECG R-peak detection using LSTM," in 35th Annual ACM Symposium on Applied Computing (New York, NY), 1104-1111. doi: 10.1145/3341105.3373945

Ma, B., Wu, Z., Li, S., Benton, R., Li, D., Huang, Y., et al. (2019). "A SVM-based algorithm to diagnose sleep apnea," in 2019 IEEE International Conference on Bioinformatics and Biomedicine (BIBM) (San Diego, CA), 1556-1560. doi: 10.1109/BIBM47256.2019.898 3201

Mahapatra, R., and Sahoo, S. (2017). "A collaborative approach to creating ictbased sustainable development," in AMCIS (Boston, MA).

Mareiniss, D. P. (2020). The impending storm: COVID-19, pandemics and our overwhelmed emergency departments. Am. J. Emerg. Med. 38, 1293-1294. doi: 10.1016/j.ajem.2020.0 3.033

Massaroni, A., Schena, A., Silvestri, S., Taffoni, F., and Merone, M. (2018). "Measurement system based on rbg camera signal for contactless breathing pattern and respiratory rate monitoring," in 2018 40th Annual International Conference of the IEEE Engineering in Medicine and Biology Society (EMBC) (Rome), 1-6. doi: 10.1109/MeMeA.2018.8438692

Mehraeen, E., Seyed Alinaghi, S. A., Nowroozi, A., Dadras, O., Alilou, S., Shobeiri, P., et al. (2020). A systematic review of ECG findings in patients with covid-19. Ind. Heart J. 72, 500-507. doi: 10.1016/j.ihj.2020.11.007

mTelehealth (2020). 2019 Medicare Physician Fee Schedule and Quality Payment Program CMS Proposed Rule CPT Codes 99453, 99454, and 99457-Everything You Need to Know. mTelehealth.

Muo, A. D. (2020). Vitalpatch Granted EUA for Monitoring COVID-19 Patients' QT INTERVALS. HIMSS.

Nosrati, M., and Tavassolian, N. (2018). High-accuracy heart rate variability monitoring using doppler radar based on Gaussian pulse train modeling and FTPR algorithm. IEEE Trans. Microw. Theory Techn. 66, 556-567. doi: 10.1109/TMTT.2017.2721407

Ohata, T., Ishibashi, K., and Sun, G. (2019). "Non-contact blood pressure measurement scheme using doppler radar," in 2019 41st Annual International Conference of the IEEE Engineering in Medicine and Biology Society (EMBC) (Berlin), 778-781. doi: 10.1109/EMBC.2019.88 57056

Pirhonen, J., Tiilikainen, E., Pekkarinen, S., Lemivaara, M., and Melkas, H. (2020). Can robots tackle late-life loneliness? Scanning of future opportunities and challenges in assisted living facilities. Futures 124:102640. doi: 10.1016/j.futures.2020.102640

Qadri, Y. A., Nauman, A., Zikria, Y. B., Vasilakos, A. V., and Kim, S. W. (2020). The future of healthcare internet of things: A survey of emerging technologies. IEEE Commun. Surv. Tutor. 22, 1121-1167. doi: 10.1109/COMST.2020. 2973314

Rangel, A., and Castillo, A. (2020). Implementing air flow sensor in a medical mask for breathing detection. Health Technol. 10, 405-410. doi: 10.1007/s12553-019-00356-x

Raza, M., Hoa Le, M., Aslam, N., Hieu Le, C., Tam Le, N., and Ly Le, T. (2018). "Telehealth technology: potentials, challenges and research directions for developing countries," in 6th International Conference on the Development of Biomedical Engineering in Vietnam (BME6). BME 2017. IFMBE Proceedings, Vol. 63, eds T. Vo Van, T. Nguyen Le, and T. Nguyen Duc (Singapore: Springer). doi: 10.1007/978-981-10-4361-1_89

Scully, A., Lee, A., Meyer, J., Gorbach, A. M., Granquist-Fraser, D., Mendelson, Y., et al. (2011). Physiological parameter monitoring from optical recordings with a mobile phone. IEEE Trans. Biomed. Eng. 59, 303-306. doi: 10.1109/TBME.2011.2163157

Shadwell, T. (2020). Robots found to improve mental health and loneliness in elderly people. Available online at: https://www.mirror.co.uk/news/uk-news/ robots-found-improve-mental-health- 22645744

Shi, A., Wang, A., Shao, A., Huang, J., Gan, J., Huang, X., et al. (2020). COVID19 infection: the perspectives on immune responses. Cell Death Differ. 27, 1451-1454. doi: 10.1038/s41418-020-0530-3

Tabacof, L., Kellner, C., Breyman, E., Dewil, S., Braren, S., Nasr, L., et al. (2020). Remote patient monitoring for home management of coronavirus disease in New York: a cross-sectional observational study. Telemed. e-Health 27. doi: $10.1089 /$ tmj.2020.0339 
Taylor, W., Abbasi, Q. H., Dashtipour, K., Ansari, S., Shah, S. A., Khalid, A., et al. (2020). A review of the state of the art in non-contact sensing for COVID-19. Sensors 20:5665. doi: 10.3390/s20195665

Tsai, Y. C., Lai, S. H., Ho, C. J., Wu, F. M., Henrickson, L., Wei, C. C., et al. (2020). "High accuracy respiration and heart rate detection based on artificial neural network regression," in 2020 42nd Annual International Conference of the IEEE Engineering in Medicine Biology Society (EMBC) (Montreal, QC), 232-235. doi: 10.1109/EMBC44109.2020.9175161

Walterscheid, I., Biallawons, O., and Berens, P. (2019). "Contactless respiration and heartbeat monitoring of multiple people using a 2-d imaging radar," in 2019 41st Annual International Conference of the IEEE Engineering in Medicine and Biology Society (EMBC) (Berlin), 3720-3725. doi: 10.1109/EMBC.2019.8856974

Wang, Y., Hu, M., Zhou, Y., Li, Q., Yao, N., Zhai, G., et al. (2020). Unobtrusive and automatic classification of multiple people's abnormal respiratory patterns in real time using deep neural network and depth camera. IEEE Internet Things J. 7, 8559-8571. doi: 10.1109/JIOT.2020.2991456

Wang, R., Jia, W., Mao, Z. H., Sclabassi, R. J., and Sun, M. (2014). "Cuff-free blood pressure estimation using pulse transit time and heart rate," in 2014 12th International Conference on Signal Processing (ICSP) (Hangzhou), 115-118. doi: 10.1109/ICOSP.2014.7014980

Wetsman, N. (2020). FDA Confirms Samsung's Galaxy Watch 3 Is Cleared for EKG, Just Like the Apple Watch. The Verge.

WHO (2016). Global Difusion of Ehealth: Making universal health coverage achievable. Licence: CC by-NC-SA 3.0 IGO. WHO.

WHO (2019). Draft Global Strategy on Digital Health 2020-2025. WHO.
WHO, A. (2020a). Coronavirus (COVID-19). WHO.

WHO, A. (2020b). COVID-19: Vulnerable and High Risk Groups. WHO.

WHO, A. (2020c). Home Care for Patients With Suspected or Confirmed COVID-19 and Management of Their Contacts. WHO.

Yang, X., Fan, D., Ren, A., Zhao, N., and Alam, M. (2019). 5G-based usercentric sensing at C-band. IEEE Trans. Indus. Inform. 15, 3040-3047. doi: 10.1109/TII.2019.2891738

Yang, X., Fan, D., Ren, A., Zhao, N., Shah, S. A., Alomainy, A., et al. (2020). Diagnosis of the hypopnea syndrome in the early stage. Neural Comput. Appl. 32, 855-866. doi: 10.1007/s00521-019-04037-8

Zheng, A., Ma, A., Zhang, J. Y., and Xie, X. (2020). COVID-19 and the cardiovascular system. Nat. Rev. Cardiol. 17, 259-260 doi: 10.1038/s41569-020-0360-5

Conflict of Interest: The authors declare that the research was conducted in the absence of any commercial or financial relationships that could be construed as a potential conflict of interest.

Copyright (C) 2021 Suresh Kumar, Dashtipour, Abbasi, Imran and Ahmad. This is an open-access article distributed under the terms of the Creative Commons Attribution License (CC BY). The use, distribution or reproduction in other forums is permitted, provided the original author(s) and the copyright owner(s) are credited and that the original publication in this journal is cited, in accordance with accepted academic practice. No use, distribution or reproduction is permitted which does not comply with these terms. 